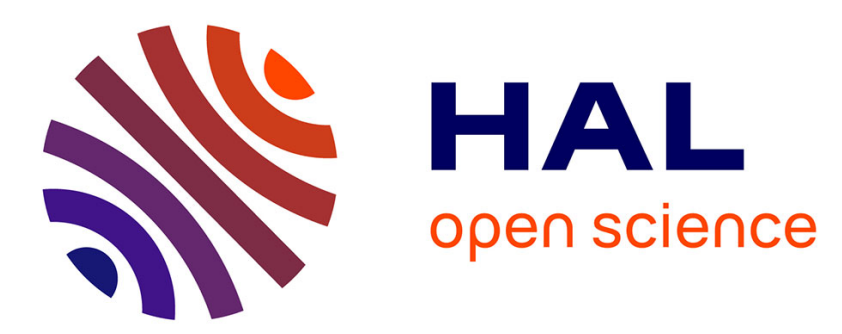

\title{
Hypothèses sur la relaxation en surface de l'hydrogène polarisé
}

\author{
M. Papoular
}

\section{To cite this version:}

M. Papoular. Hypothèses sur la relaxation en surface de l'hydrogène polarisé. Journal de Physique Lettres, 1984, 45 (3), pp.137-141. 10.1051/jphyslet:01984004503013700 . jpa-00232320

\section{HAL Id: jpa-00232320 https://hal.science/jpa-00232320}

Submitted on 1 Jan 1984

HAL is a multi-disciplinary open access archive for the deposit and dissemination of scientific research documents, whether they are published or not. The documents may come from teaching and research institutions in France or abroad, or from public or private research centers.
L'archive ouverte pluridisciplinaire HAL, est destinée au dépôt et à la diffusion de documents scientifiques de niveau recherche, publiés ou non, émanant des établissements d'enseignement et de recherche français ou étrangers, des laboratoires publics ou privés. 
Classification

Physics Abstracts

$67.70-67.90-34.90$

\title{
Hypothèses sur la relaxation en surface de l'hydrogène polarisé
}

\author{
M. Papoular \\ CNRS/CRTBT, BP 166 X, 38042 Grenoble, France
}

(Reçu le 25 août 1983, révisé le 15 novembre, accepté le ler décembre 1983)

\begin{abstract}
Résumé. - Les caractéristiques paradoxales de la relaxation de surface déterminée expérimentalement sont brièvement discutées. Une voie possible de dépolarisation par chocs consécutifs à la recombinaison en surface est proposée. Ce canal préserve la dépendance en champ magnétique et élimine l'anisotropie caractéristique de la relaxation dipolaire.
\end{abstract}

Abstract. - The paradoxal observed properties of surface relaxation are briefly discussed. A possible depolarization channel through collisions with freshly recombined molecules is described. This would explain the magnetic field dependence and eliminate the anisotropy associated with dipolar surface relaxation.

1. - Il est maintenant acquis que la relaxation $T_{1}$ en volume de $\mathrm{H}$ atomique polarisé, sous champ magnétique fort, est gouvernée par l'interaction dipolaire, ce qui lui confère une dépendance caractéristique en champ magnétique $[1,2]$ :

$$
T_{1}^{-1}=2 n G_{\mathrm{v}} \sim\left(1+\frac{16,7}{B}\right)^{2}
$$

$B$ étant compté en tesla. $T_{1}$ est le temps, $G$ le taux de relaxation, $n$ la densité volumique d'atomes $\mathrm{H} \downarrow$.

La relaxation en surface $G_{s}$, elle, n'est pas comprise [3]. Elle est signée par une dépendance en $T$ effectivement exponentielle, semble-t-il $[1,2,4]$, sur la plage linéaire de l'isotherme d'adsorption : $G_{\mathrm{s}}^{\text {eff }} \sim T^{-1} \exp 2 \varepsilon_{\mathrm{a}} / T$, où $\varepsilon_{\mathrm{a}}$ est l'énergie d'adsorption $(\simeq 1 \mathrm{~K}$, pour un revêtement $\mathrm{d}^{\mathbf{4}} \mathrm{He}$ liquide). Elle présente la même dépendance en $B$ que $G_{\mathrm{v}}$, alors même que, sur ${ }^{4} \mathrm{He}$, (i) $\left(G_{\mathrm{s}}\right)_{\exp } \sim 50\left(G_{\mathrm{s}}\right)_{\mathrm{calc}}^{\mathrm{dipol}}$; (ii) $\left(G_{\mathrm{s}}\right)_{\exp }$ ne semble pas présenter l'anisotropie caractéristique de $\left(G_{\text {s }}\right)_{\text {calc }}^{\text {dipol }}$. Enfin, un revêtement $d^{3}{ }^{3} \mathrm{He} \mathrm{liquide} \mathrm{relaxe} \mathrm{beaucoup} \mathrm{moins} \mathrm{que}{ }^{4} \mathrm{He}$; en fait $\left(G_{\mathrm{s}}\right)_{\text {exp }}$ sur ${ }^{3} \mathrm{He}$ est nul aux incertitudes près, au moins jusqu'à 100 ou $80 \mathrm{mK}$ (Réf. [1]; il faut remarquer que cette expérience est (à densité forte) la seule jusqu'ici à utiliser un revêtement d' ${ }^{3} \mathrm{He}$ ); en particulier, $\left(G_{\mathrm{s}}\right)_{\exp { }^{3} \mathrm{He}} \ll\left(G_{\mathrm{s}}\right)^{\text {dipol }}$ calculé pour une orientation oblique par rapport au champ (la paroi étant essentiellement $\perp \mathbf{B}$ dans [1], ce résultat (pour ${ }^{3} \mathrm{He}$ ) est cohérent avec l'anisotropie $\left.\operatorname{de}\left(G_{\mathrm{s}}\right)_{\mathrm{calc}}^{\text {dipol }}\right)$.

Le fait que $\left(G_{\mathrm{s}}\right)_{\exp }$ ait la même dépendance en $B$ que $G_{\mathrm{v}}$ nous conduit à relier relaxation en surface et en volume, en tenant compte de ce que le taux de recombinaison (recombinaison en surface) est commandé par $G_{\mathrm{v}}$ (puisque $\left.G_{\mathrm{v}} \ll K_{\mathrm{s}}^{\text {eff }}[4,1]\right)$ : si chaque recombinaison, libérant un «projectile » $\mathbf{H}_{2}$, s'accompagne d'une certaine probabilité de dépolarisation en volume, 
on retrouve bien $G_{\mathrm{s}}^{\text {eff }} \sim G_{\mathrm{v}} \sim\left(1+\frac{16,7}{B}\right)^{2}$. Dans la section suivante, nous décrivons un mécanisme possible pour cet enchaînement : $G_{\mathrm{v}} \rightarrow K_{\mathrm{s}}^{\text {eff }} \rightarrow G_{\mathrm{s}}^{\text {eff }}$. En ce qui concerne le résultat négatif sur ${ }^{3} \mathrm{He}$, il est clair que, avec un profil en marche d'escalier pour l'interface He/vide, un tel effet " isotopique » est difficile à justifier : après tout. le rapport des énergies d'adsorption sur ${ }^{4} \mathrm{He}$ et ${ }^{3} \mathrm{He}$ n'est que de 2,5. Par contre, si nous admettons que le profil ${ }^{3} \mathrm{He} / v i d e$ est moins brutal, on conçoit qu'une fraction beaucoup plus importante de l'énergie libérée par chaque recombinaison soit dissipée dans $1{ }^{3} \mathrm{He}$ lui-même et ne soit donc plus disponible pour entraîner une relaxation ultérieure. On dispose d'une théorie de l'interface ${ }^{4} \mathrm{He} /$ vide [5], mais non pas pour ${ }^{3} \mathrm{He}$. On peut penser que, du fait à la fois de sa plus grande énergie cinétique de point zéro, et de la répulsion de Pauli, l' ${ }^{3} \mathrm{He}$ tend à "étaler " sa surface libre sur une plus longue distance (à rapprocher du fait que la chaleur de vaporisation est trois fois plus faible pour ${ }^{3} \mathrm{He}$ ). Ce point mériterait sans doute une étude en soi, indépendamment du problème de $G_{\mathrm{s}}$. Nous nous contenterons ici d'avancer l'hypothèse.

Remarquons que, parmi les interprétations qu'on a pu proposer pour comprendre la relaxation de surface, deux paraissent a priori séduisantes : (i) la surface d'He déforme l'atome d'hydrogène (comme l'atteste d'ailleurs le déplacement correspondant de la fréquence de Larmor [6]), lui conférant une petite amplitude de désorientation de spin [3]. Mais ce processus ne permet de comprendre ni la dépendance en $B$, ni l'effet "isotopique "; (ii) à 2 dimensions, la formation de paires $\mathrm{H} \downarrow-\mathrm{H} \downarrow$, de courte durée de vie est favorisée (voir par ex. Réf. [7]); du fait de leur faible rayon, ces paires relaxeraient, par couplage dipolaire, plus efficacement : on comprend alors $f(B)$ mais l'effet isotopique est plus difficile à interpréter quantitativement - encore que la moindre énergie d'adsorption conduise à une relaxation plus faible en effet sur ${ }^{3} \mathrm{He}$.

2. - Le mécanisme de relaxation que nous invoquons ici implique (via le couplage spin-orbite) la déformation de la fonction d'onde orbitale de l'atome d'hydrogène, consécutive à une collision. L'amplitude de diffusion pour 2 particules de spin 1/2 doit être un scalaire invariant par renversement du temps. La forme la plus générale en est [8] :

$f=A+B\left(\mathbf{s}_{1} \cdot \lambda\right)\left(\mathbf{s}_{2} \cdot \lambda\right)+C\left(\mathbf{s}_{1} \cdot \boldsymbol{\mu}\right)\left(\mathbf{s}_{2} \cdot \mu\right)+D\left(\mathbf{s}_{1} \cdot v\right)\left(\mathbf{s}_{2} \cdot v\right)+E\left(\mathbf{s}_{1}+\mathbf{s}_{2}\right) \cdot v+F\left(\mathbf{s}_{1}-\mathbf{s}_{2}\right) \cdot v$

où $\lambda, \mu$ et $\boldsymbol{v}$ sont trois vecteurs unitaires, mutuellement orthogonaux, orientés par rapport aux directions d'incidence et de diffusion. Les coefficients $A, B$ etc. ne dépendent que de l'angle de diffusion et, surtout, de l'énergie. Une estimation du taux de dépolarisation a été faite [9] dans le cas plus simple où la particule-projectile ne porte pas de spin $(f$ se réduit alors à $: \alpha+\beta \mathbf{s . v})$. Pour l'hydrogène polarisé, l'interaction a lieu dans l'état triplet où la distance minimum d'approche est grande aux énergies thermiques ( 7 fois le rayon de Bohr) : pour des collisions thermiques. la déformation orbitale est donc négligeable, et l'effet aussi.

Mais nous avons en vue le cas plus intéressant d'un choc énergétique. A la suite du bilan de recombinaison, une molécule $\mathrm{H}_{2}$ est animée d'une forte énergie cinétique dont elle communiquera une fraction importante $(\sim 2 / 3)$ aux atomes $\mathrm{H} \downarrow$ qu'elle heurtera $: \sim 100 \mathrm{~K}$ à la formation de $\mathrm{H}_{2}$ dans l'état de vibration-rotation $v=14, l=3$; et, surtout, $\sim 10^{4} \mathrm{~K}$ par désexcitation vers le fondamental de l'état singulet. A leur tour, ces atomes $\mathbf{H}$ " chauds » entreront en collision avec d'autres $\mathrm{H} \downarrow$, etc., selon une cascade énergétique 2 fois moins intense mais 2 fois plus dense à chaque " génération ".

On montre [9] qu'un coefficient de couplage tel que $\beta$ est proportionnel au carré du taux d'hybridation $\Lambda$ induit par la collision (hybridation $\mathrm{P}$ en particulier) :

$$
\Lambda\left(b_{0}\right) \simeq-\frac{E_{\text {int }}\left(b_{0}\right)}{E_{\mathrm{at}}} \frac{b_{0}}{a_{0}},
$$


$\left(a_{0}\right.$, rayon de Bohr $; b_{0}$, distance d'approche : $E_{\mathrm{at}}$, énergie d'un niveau atomique excité (quelques $\mathrm{eV}) ; E_{\text {int }}$ interaction collisionnelle). Dans notre cas, $E_{\text {int }}$ est fixé par l'énergie cinétique : $\Lambda(1 \mathrm{~K}) \sim$ $10^{-4}, \Lambda\left(10^{2} \mathrm{~K}\right) \sim 10^{-2}$. La procédure variationnelle [9] qui conduit à cette expression de $\Lambda$ interdit de l'extrapoler jusqu'à $E_{\text {int }} \sim 10^{4} \mathrm{~K}$. On voit cependant que, à $10^{2} \mathrm{~K}$ déjà, la section efficace de dépolarisation, qui est proportionnelle à $\beta^{2} \sim \Lambda^{4}$. est supérieure de plusieurs ordres de grandeur à ce qu'elle serait aux énergies thermiques. Nous nous bornerons donc à suggérer que l'effet est important, d'autant qu'une seule recombinaison donne lieu à plusieurs séquences énergétiques à mesure de la désexcitation de $\mathrm{H}_{2}$. Il s'agit bien d'une "relaxation de surface " dans la mesure où la recombinaison primaire a lieu en surface.

Dès lors, l'équation d'évolution pour les populations des deux niveaux hyperfins les plus bas de H, s'écrit simplement :

$$
\begin{gathered}
\dot{n}_{\mathrm{a}}=G_{\mathrm{v}} n_{\mathrm{b}}^{2}-K_{\mathrm{s}}^{\text {eff }} n_{\mathrm{a}} n_{\mathrm{b}} \\
\dot{n}_{\mathrm{b}}=-G_{\mathrm{v}} n_{\mathrm{b}}^{2}-K_{\mathrm{s}}^{\text {eff }} n_{\mathrm{a}} n_{\mathrm{b}}-p K_{\mathrm{s}}^{\text {eff }} n_{\mathrm{a}} n_{\mathrm{b}}^{2} .
\end{gathered}
$$

Le premier terme représente la relaxation en volume, le deuxième la recombinaison en surface (traduite en termes de densités volumiques : voir par ex. [1]); le troisième terme dans ( $3 b$ ) représente l'effet de relaxation effective en surface que nous venons de décrire qualitativement. Noter que l'efficacité $p$ du processus a les dimensions $L^{3}$. L'effet bien connu de distillation dans l'état b résulte de $(3 a)$ :

$$
\frac{n_{\mathrm{a}}}{n_{\mathrm{b}}}=\frac{G_{\mathrm{v}}}{K_{\mathrm{s}}^{\text {eff }}} \ll 1 .
$$

Pour simplifier, nous nous limiterons à une relaxation de surface assez modeste pour que cette inégalité forte reste vérifiée. C'est d'ailleurs à cette condition que l'équation d'évolution prend la forme simple (3), car la densité d'atomes dépolarisés reste faible devant $n_{\mathrm{a}}$. L'équation d'évolution dans le cas général ainsi qu'une estimation de la probabilité de dépolarisation (voir fin sect. 2), seront données dans un travail plus détaillé.

Venons-en au terme

$$
p K_{\mathrm{s}}^{\text {eff }} n_{\mathrm{a}} n_{\mathrm{b}}^{2}
$$

dans (3b). Le produit $\left(K_{\mathrm{s}}^{\text {eff }} n_{\mathrm{a}} n_{\mathrm{b}}\right)$ y représente le flux de molécules $\mathrm{H}_{2}$-projectiles. La densité d'atomes $\mathrm{H}$ dépolarisés par collision est donc proportionnelle à $K_{\mathrm{s}}^{\text {eff }} n_{\mathrm{a}} n_{\mathrm{b}}$. Faisant l'approximation habituelle d'une cinétique rapide d'adsorption-désorption (qui préserve la relation d'équilibre $n_{\text {surf }}=n_{\text {vol }} \lambda_{\mathrm{B}} \exp \frac{\varepsilon_{\mathrm{a}}}{T}$, où $\lambda_{\mathrm{B}} \sim \frac{1}{\sqrt{T}}$ est la longueur d'onde thermique de de Broglie), il en résulte que la densité adsorbée de ces atomes dépolarisés est proportionnelle à $\left(K_{\mathrm{s}}^{\text {eff }} n_{\mathrm{a}} n_{\mathrm{b}}\right) T^{-1 / 2} \exp \frac{\varepsilon_{\mathrm{a}}}{T}$. Ils sont maintenant prêts à recombiner avec des atomes $b$ déjà adsorbés, de densité également proportionnelle à $T^{-1 / 2} \exp \frac{\varepsilon_{\mathrm{a}}}{T}$, ce qui finalement apparaîtra comme une relaxation. D'où, dans l'expression (5), le facteur $n_{\mathrm{b}}^{2}$ et un facteur $T^{-1} \exp 2 \frac{\varepsilon_{\mathrm{a}}}{T}$ que nous incluons dans $p$ :

$$
p=p_{0} T^{-1} \exp 2 \frac{\varepsilon_{\mathrm{a}}}{T}
$$

Cela précisé, l'équation (3) se résout immédiatement ; compte tenu de (4) :

$$
-\frac{\dot{n}_{\mathrm{b}}}{n_{\mathrm{b}}}=T_{1}^{-1}=2 n_{\mathrm{b}} G_{\mathrm{v}}+p n_{\mathrm{b}}^{2} G_{\mathrm{v}} .
$$


On voit que, à mesure que la température baisse, se superpose à la relaxation volumique $2 n_{\mathrm{b}} G_{\mathrm{v}}$. un terme de surface :

$$
G_{\mathrm{s}}^{\mathrm{eff}}=\frac{1}{2} G_{\mathrm{v}} p n_{\mathrm{b}}
$$

qui a la bonne dépendance en $B$ (Eq. (1)) et en $T$ (Eq. (6)), plus une dépendance " non linéaire ", en $n_{\mathrm{b}}$, que les expériences actuelles ne paraissent pas permettre de vérifier (ou d'infirmer). Nous pouvons montrer qu'une probabilité moyenne $\bar{\delta}$ de dépolarisation de l'ordre de $10^{-8}$ par collision explique le taux de relaxation expérimental. Nous écrivons pour cela que les molécules $\mathrm{H}_{2}$ fraîchement recombinées ont une probabilité, en $\mathrm{cm}^{-3} \mathrm{~s}^{-1}$, de frapper un premier atome $\mathrm{H} \downarrow$ dans le gaz, donnée par $\sigma \phi_{\mathrm{H}_{2}} n_{\mathrm{b}}$, où $\sigma$ est une section efficace cinétique $\simeq 10^{-15} \mathrm{~cm}^{2}$ et $\phi_{\mathrm{H}_{2}}=\frac{V}{A} K_{\mathrm{s}}^{\text {eff }} n_{\mathrm{a}} n_{\mathrm{b}}$, le flux de molécules quittant la surface ( $V$ volume, $A$ superficie). $\bar{\delta}$ est obtenu par comparaison avec $G_{\mathrm{s}}^{\text {eff }}$, en tenant compte de l'ensemble de la cascade de collision-dépolarisation. Ce résultat s'accorde raisonnablement avec les ordres de grandeur suggérés, dans un autre contexte (collisions $\mathrm{Rb}$-gaz rare, à basse énergie), dans la référence [9]. Ici, l'atome d'hydrogène est certes beaucoup plus léger, mais la collision est plus efficace parce que plus énergétique $\left(\delta \sim E_{\text {cin }}^{4}\right)$.

3. - En conclusion, nous proposons un mécanisme de relaxation effective en surface gouverné par la recombinaison, donc par la relaxation en volume, ce qui préserve la dépendance en champ magnétique et élimine l'anisotropie caractéristique de $\left(G_{\mathrm{s}}\right)^{\text {dipol }}$. Nos deux principales conjectures restent ouvertes : (i) sur l'efficacité du mécanisme lui-même; (ii) sur l'étalement du profil de $1^{3} \mathrm{He}$ liquide, invoqué pour expliquer l'inocuité de ${ }^{3} \mathrm{He}$ vis-à-vis de la relaxation de surface. Il est clair que de nouvelles expériences seraient bienvenues : (i) pour confirmer l'effet isotopique : (ii) pour affiner la connaissance de $G_{\mathrm{s}}(T)$, en particulier aux plus basses températures; (iii) pour rechercher une éventuelle dépendance en densité volumique - ce qui fournirait un test « pointu » à l'argument avancé ici.

En tout état de cause, il est acquis d'une part que ${ }^{3} \mathrm{He}$ (flottant sur ${ }^{4} \mathrm{He}$ superfluide [1]) est le substrat idéal puisque $\mathrm{H} \downarrow$ y relaxe beaucoup moins, et qu'il transmet mieux la chaleur [10], et d'autre part que, sur ${ }^{4} \mathrm{He}, G_{\mathrm{s}}$ domine à basse température.

Ces considérations sont déterminantes quant aux conditions d'accès à la condensation de Bose. Nous terminerons sur deux remarques relatives aux fortes densités volumiques nécessaires à l'observation de cette condensation.

a) Lorsque $n$ augmente, un nouveau canal de relaxation entre en jeu : la relaxation-recombinaison à 3 particules (d'origine dipolaire encore [11]), récemment observée [12, 13]. C'est à basse température, toujours, qu'il sera le plus dangereux : $n^{\mathrm{s}}>10^{12} \mathrm{~cm}^{-2}$, relaxation en surface; ce processus toutefois, comme la relaxation dipolaire à 2 particules en surface, est anisotrope et disparaît sur une surface strictement perpendiculaire à $B$. On peut donc espérer s'en affranchir avec une cellule très plate (l'une des solutions retenues par l'équipe de Grenoble).

b) On pourrait craindre malgré tout qu'une compression, même rapide, en raccourcissant le temps $T_{1}$ en volume (dans la proportion du taux de compression), augmente de façon prohibitive la chaleur de recombinaison $\dot{Q}$. Mais dans la mesure où, au départ, on est déjà à $T$ assez faible et $n$ assez grand pour se trouver sur le plateau de l'isotherme d'adsorption $\left(n^{\mathrm{s}} \simeq\left(n^{\mathrm{s}}\right)_{\max }\right)$, tout accroissement de $\dot{Q}$ requiert la transformation d'atomes b en atomes a, c'est-à-dire précisément un temps $T_{-1}$. Si $T_{1}$ reste très supérieur au temps de compression, $\dot{Q}$ ne sera pas plus dangereux immédiatement après la compression, qu'avant. 


\section{Remerciements.}

Je tiens à remercier mes collègues A. Greenberg, B. Hébral et J. B. Robert pour des discussions nombreuses et fructueuses.

\section{Bibliographie}

[1] Sprik, R., Walraven, J. T. M., van Yperen, G. H. et Silvera, I. F., Phys. Rev. Lett. 49 (1982) 153.

[2] Yurke, B., Denker, J. S., Johnson, B. R., Bigelow, N., Lévy, L. P., Lee, D. M. et Freed, J. H., Phys. Rev. Lett. 50 (1983) 1137.

[3] v. D. EiJnde, J. P., Reuver, C. J. et VerhaAR, B. J., à paraître.

[4] Cline, R. W., Greytak, T. J. et Kleppner, D., Phys. Rev. Lett. 47 (1981) 1195.

[5] Mantz, I. B. et Edwards, D. O., Phys. Rev. B 20 (1979) 4518.

[6] Jochemsen, R., Morrow, M., Berlinsky, A. J. et Hardy, W. N., Phys. Rev. Lett. 47 (1981) 852.

[7] Papoular, M., J. Low Temp. Phys. 50 (1983) 253.

[8] Landau, L. D. et Lifshitz, E. M., Quantum Mechanics, 3e éd., (Pergamon Press) 1977, p. 579.

[9] Herman, R. M., Phys. Rev. A 136 (1964) 1576.

[10] Castaing, B. et Papoular, M., J. Physique Lett. 44 (1983) L-537.

[11] Kagan, Yu. Vartanyants, I. A. et Shlyapnikov, G. V., Sov. Phys. J.E.T.P. 54 (1982) 590.

[12] Hess, H. F., Bell, D. A., Kochanski, G. P., Cline, R. W., Kleppner, D. et Greytak, T. J., Phys. Rev. Lett. 51 (1953) 483.

[13] Sprik. R., Walraven, J. T. M. et Silvera, I. F., 51 (1983) 479. 